\title{
Analyzing medwatch data in an effort to assess dronabinol post-marketing safety
}

\section{Introduction}

Safety data from the Medwatch system on all drug products from around the world can be used in pharmacovigilance to detect signals through the statistical noise. Analysis of top drug adverse event reactions from this data can be used to retrospectively assess safety in real-time. ${ }^{1,2}$ For medical marijuana, also known as dronabinol, this can be used to assure healthcare providers of its low adverse event profile. This experiment aims to evaluate the intensity of the most common adverse events reported in the drug database with all dronabinol reports with dronabinol and its reaction, all reports with the drug and no reaction, all reports with the reaction and no drug, and all other reports. From this, a Proportional Reporting Ratio will be generated, allowing for subjective and objective comparison of relative intensities.

\section{Methods}

Since 2004, publicly available MedWatch data without personal information allows the researchers to avoid Health Information Privacy violations. Physicians, pharmacists, or manufacturer's generated reports contains a number that coincides each occasion a drug was reported along with its reported reactions and a standardized one-phrase description of the reaction coordinated with this number In order to assess all dronabinol-adverse event strengths, the statistical method chosen was the Proportional Reporting Ratio, which is calculated as; $\mathrm{PRR}=\mathrm{A} /(\mathrm{A}+\mathrm{B}) / \mathrm{C} /(\mathrm{C}+\mathrm{D})$. $\mathrm{A}$ is the number of reports where a patient was on the med and experienced the Adverse Drug Event, B is the number of reports where a patient was not on the drug and experienced the Adverse Event, $\mathrm{C}$ is the number of reports where the patient was on the drug but did not experience the Adverse Event, and $\mathrm{D}$ is the number of reports where the patient was not on the drug and did not experience the Adverse Event. Once the top dronabinolAdverse event PRR was gathered for each year, comparisons can be drawn. ${ }^{2}$ Only reactions seen more than once with dronabinol use were tested in this study.

\section{Results}

The top reported dronabinol reactions from 2004 to 2015 include pancreatic carcinoma, anemia, pruritic rash, gi bleeding, back pain, joint stiffness, and pyrexia. The rate of patients who were exposed to the medication and experienced an adverse event ranged from 0.07 to 0.86 . Relative ratios should show dronabinol's relationship to each particular adverse event. The results are charted below Figure 1.

\section{Conclusion}

Although the incidence of all dronabinol adverse events is low throughout this database's history, these results highlight a use of the MedWatch data for future medical marijuana studies. Developing a system of post-marketing surveillance around the parameters in this study combined with clinical characteristics and patient notes could provide enough content to show a true correlation between different types of dronabinol administration and different adverse event rates. This particular study is limited by the severity of reactions not deemed
Volume 2 Issue 2 - 2017

\author{
Zacharya Piracha, Rohan Ramnarain \\ Department of pharmaceutical sciences, St. John's University \\ College of Pharmacy and Health Sciences, USA
}

\begin{abstract}
Correspondence: Rohan Ramnarain, Department of pharmaceutical sciences, St. John's University College of Pharmacy and Health Sciences, USA, Tel 9548260403 , Email rohan.ramnarain@gmail.com
\end{abstract}

Received: March 17, 2017| Published: March 22, 2017

high enough for reporting in this particular MedWatch collection of safety data.

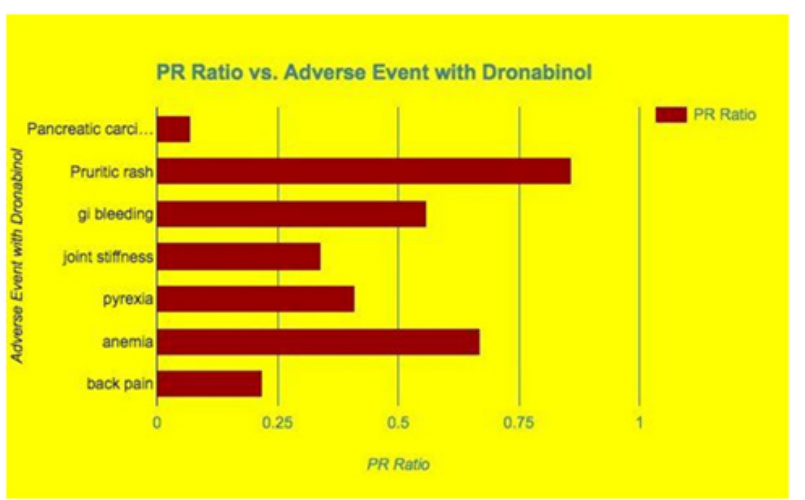

Figure I Relative ratios should show dronabinol's relationship to each particular adverse event.

Ideally, studies such as these have more clinical relevance in a field including cannabis where different strains and subtypes are known to elicit different effects on the CBD and THC associated receptors. Adverse Events could also include tolerance and down regulation of receptors over time, as well as the incidence of new drug interactions when new strains emerge. These studies are not fully supported by the federal standards but as more organizations legitimize the use of medical marijuana for sizeable clinical benefits, we can also hope to regulate this budding industry through a pharmaceutical drug safety perspective, armed with scientific and statistical models. Those evaluating the validity of conclusions drawn from this report should consider the Proportional Reporting Ratio intervals not strong enough to warrant concern but also not indicative of any no inferiority in terms of safety. The adverse events linked to dronabinol in this database may very well over time demonstrate a strong enough signal to cause federal concern, something future studies must address. ${ }^{3}$

\section{Acknowledgements}

None.

\section{Conflict of interest}

The author declares no conflict of interest. 


\section{References}

1. Ali AK. Pharmacovigilance analysis of serious adverse events reported for biologic response modifiers used as prophylaxis against transplant rejection: a real-world post marketing experience from the US FDA adverse event reporting system (FAERS). Int J Organ Transplant Med. 2013;4(2):62-71.
2. Evans SJ, Waller PC, Davis S. Use of proportional reporting ratios (PRRs) for signal generation from spontaneous adverse drug reaction reports. Pharmacoepidemiol Drug Saf. 2001;10(6):483-486.

3. Hauben M, Patadia V, Gerrits C, et al. Data mining in pharmacovigilance: the need for a balanced perspective. Drug Saf. 2005;28(10):835-842. 